Pacific

Journal of

Mathematics

\author{
A PRESENTATION \\ FOR THE AUTOMORPHISMS OF THE 3-SPHERE \\ THAT PRESERVE A GENUS TWO HEEGAARD SPLITTING
}

EROL AKBAS

Volume $236 \quad$ No. 2

June 2008 


\title{
A PRESENTATION \\ FOR THE AUTOMORPHISMS OF THE 3-SPHERE THAT PRESERVE A GENUS TWO HEEGAARD SPLITTING
}

\author{
EROL AKBAS
}

\begin{abstract}
Scharlemann constructed a connected simplicial 2-complex $\Gamma$ with an action by the group $\mathscr{H}_{2}$ of isotopy classes of orientation-preserving homeomorphisms of $S^{3}$ that preserve the isotopy class of an unknotted genus 2 handlebody $V$. In this paper we prove that the 2 -complex $\Gamma$ is contractible. Therefore we get a finite presentation of $\mathscr{H}_{2}$.
\end{abstract}

\section{Introduction}

Let $\mathcal{H}_{g}$ be the group of isotopy classes of orientation-preserving homeomorphisms of $S^{3}$ that preserve the isotopy class of an unknotted genus $g$ handlebody $V$. In [1933], Goeritz proved that $\mathscr{H}_{2}$ is finitely generated. In 1977, Goeritz's theorem was generalized to arbitrary genus $g \geq 2$ by Jerome Powell [1980]. In 2003, Martin Scharlemann noticed that Powell's proof contains a serious gap. Scharlemann [2004] gave a modern proof of Goeritz's theorem by introducing a simplicial 2complex $\Gamma$, with an action by $\mathscr{H}_{2}$, that deformation retracts onto a graph $\tilde{\Gamma}$. Given any two distinct vertices $v, \tilde{v}$ of $\Gamma$, Scharlemann constructed a vertex $u$ in $\Gamma$ that is adjacent to $v$ and "closer" to $\tilde{v}$ (by "closer" we mean the intersection number of $u$ and $\tilde{v}$; see Definition 1). Hence $\mathscr{H}_{2}$ acts on the connected graph $\tilde{\Gamma}$ and is generated by the isotopy classes of elements denoted by $\alpha, \beta, \gamma$, and $\delta$ (see Section 2 for a complete description). In this paper we study the geometry of $\Gamma$ by showing that $u$ is essentially unique (for a precise statement see Proposition 2). We derive the following theorem.

Theorem 1. The graph $\tilde{\Gamma}$ is a tree, and shortest paths can be calculated algorithmically.

Note that $\tilde{\Gamma}$ is locally infinite. So calculating paths is not trivial. We also get

Theorem 2. (i) $\mathscr{H}_{2}$ has generators $[\alpha],[\beta],[\gamma]$, and $[\delta]$ and relations $[\alpha]^{2}=$ $[\gamma]^{2}=[\delta]^{3}=[\alpha \gamma]^{2}=\left[\alpha \delta \alpha \delta^{-1}\right]=\left[\alpha \beta \alpha \beta^{-1}\right]=1,[\gamma \beta \gamma]=[\alpha \beta]$, and $[\delta]=$ $\left[\gamma \delta^{2} \gamma\right]$.

MSC2000: primary 57M60; secondary 57M20.

Keywords: Heegaard, genus two handlebody, three-sphere. 
(ii) $\mathscr{H}_{2} \cong\left(\mathbb{Z} \oplus \mathbb{Z}_{2}\right) \rtimes \mathbb{Z}_{2} \underset{\mathbb{Z}_{2} \oplus \mathbb{Z}_{2}}{*}\left(\mathbb{Z}_{3} \rtimes \mathbb{Z}_{2}\right) \oplus \mathbb{Z}_{2}$

\section{Preliminaries}

We give a description of the 2-complex $\Gamma$ introduced in [Scharlemann 2004], to which we refer for details about $\Gamma$.

Let $V$ be an unknotted handlebody of genus two in $S^{3}$, and let $W$ be the closure of its complement. Let $T$ be the boundary of $V$. Then $T$ is a genus two Heegaard surface for $S^{3}$. Let $\mathscr{H}_{2}$ denote the group of isotopy classes of orientation-preserving homeomorphisms of $S^{3}$ that leave the genus two handlebody $V$ invariant. A sphere $P$ in $S^{3}$ is called a reducing sphere for $T$ if $P$ intersects $T$ transversely in a simple closed curve which is homotopically nontrivial on $T$. For any reducing sphere $P$ for $T$, let $c_{P}$ denote $P \cap T$, and let $v_{P}$ denote the isotopy class of $c_{P}$ on $T$.

Definition 1. For any two reducing spheres $R, Q$ for $T$, define the intersection number of $v_{R}$ and $v_{Q}$ as

$$
v_{R} \cdot v_{Q}=\min _{\substack{c_{R^{\prime}} \in v_{R} \\ c_{Q^{\prime}} \in v_{Q}}}\left|c_{R^{\prime}} \cap c_{Q^{\prime}}\right|
$$

where $\left|c_{R^{\prime}} \cap c_{Q^{\prime}}\right|$ is the geometric intersection number of $c_{R^{\prime}}$ with $c_{Q^{\prime}}$.

Let $\Gamma$ be a complex whose vertices are isotopy classes of reducing spheres for $T$. A collection $P_{0}, \ldots, P_{n}$ of reducing spheres bounds an $n$-simplex in $\Gamma$ if and only if $v_{P_{i}} \cdot v_{P_{j}}=4$ for all $0 \leq i \neq j \leq n$. In fact $n \leq 2$; see [Scharlemann and Thompson 2003, Lemma 2.5]. So $\Gamma$ is a simplicial 2-complex. See Figure 1 for a local picture of $\Gamma$ and a picture of three curves forming the vertices of a 2-simplex in $\Gamma$. Let $\triangle$ be any 2-simplex of $\Gamma$. We denote by $S_{\triangle}$ the "spine" of $\triangle$, which is the subcomplex of the barycentric subdivision consisting of all closed 1-simplices that contain the barycenter and a vertex of $\Delta$. Clearly $\Delta$ deformation retracts onto $S_{\triangle}$. Let

$$
\tilde{\Gamma}=\bigcup_{\triangle} S_{\triangle}
$$

So $\tilde{\Gamma}$ is a graph. Since no two 2-simplices of $\Gamma$ share an edge [Scharlemann and Thompson 2003, Lemma 2.5], the simplicial 2-complex $\Gamma$ deformation retracts onto the graph $\tilde{\Gamma}$.

A belt curve on a genus two surface is a homotopically nontrivial separating simple closed curve. Let $P$ denote a reducing sphere whose intersection with $T$ is a belt curve, which we denote $c_{P}$. The reducing sphere $P$ divides $S^{3}$ into two 3-balls $B^{ \pm}$whose intersections with the genus two surface $T$ are two genus one surfaces $T^{ \pm}=T \cap B^{ \pm}$, each having one boundary component. The surface $T^{-}$ (respectively $T^{+}$) contains two simple closed curves $B, Z$ (respectively $C, Y$ ) 

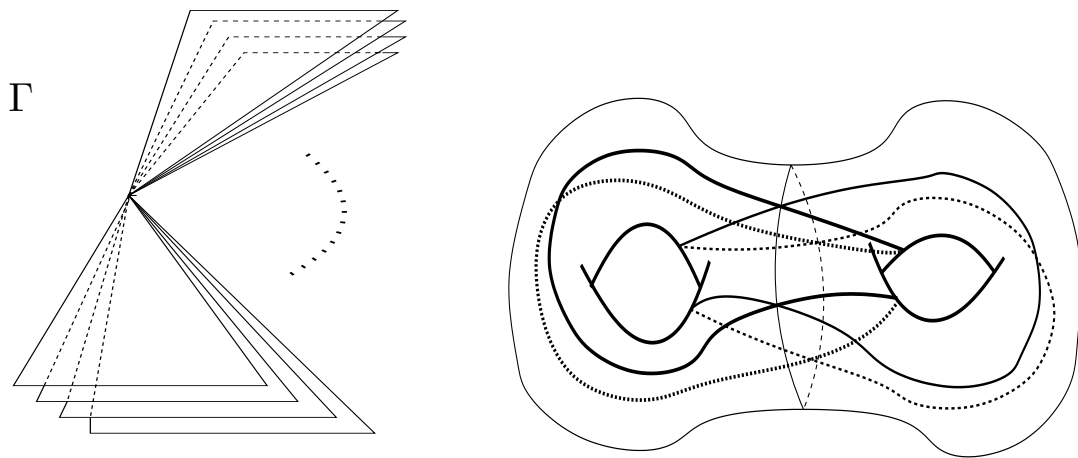

Figure 1. Left: locally $\Gamma$. Right: three curves forming the vertices of a 2 -simplex in $\Gamma$.

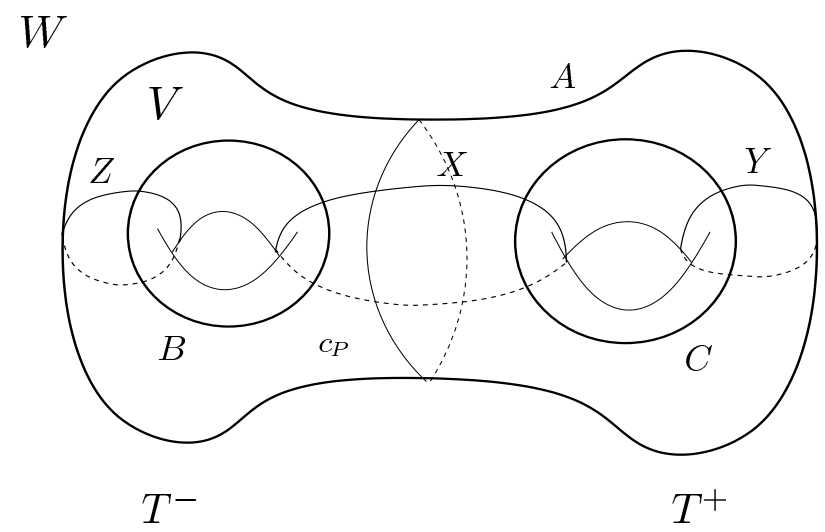

Figure 2. The curves $c_{P}, A, B, C, X, Y$, and $Z$.

meeting at one point. The curve $B$ (respectively $C$ ) bounds a nonseparating disc in $W$ which is homotopically nontrivial in $V$. The curve $Z$ (respectively $Y$ ) bounds a nonseparating disc in $V$ which is homotopically nontrivial in $W$. The genus two surface $T$ contains two disjoint simple closed curves $A$ and $X$. The curve $A$ is homotopically nontrivial in $V$, disjoint from $B$ and $C$, bounds a nonseparating disc in $W$, and intersects $Z$ and $Y$ at one point. The curve $X$ is homotopically nontrivial in $W$, disjoint from $Z, Y$ and $A$, bounds a nonseparating disc in $V$, and intersects $B$ and $C$ at one point. See Figure 2.

Throughout this paper, unless otherwise stated, whenever we choose a reducing sphere $R$ for $T$ such that $v_{R} \neq v_{P}$, we will assume that the curve $c_{R}$ intersects $c_{P}$, $B, C, Y, Z$ transversely and minimally and intersects $A$ transversely. 

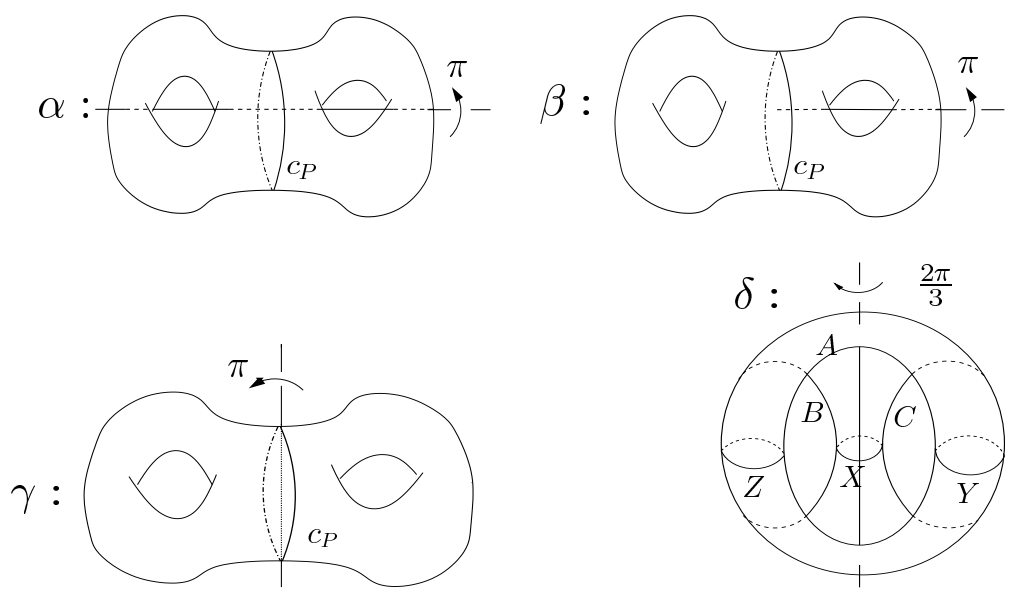

Figure 3. Homeomorphisms $\alpha, \beta, \gamma$ and $\delta$.

There exist three automorphisms $\alpha, \beta, \gamma$ of $S^{3}$ with the following properties. The automorphism $\alpha$ is an orientation-preserving homeomorphism of $S^{3}$ that preserves $V$ and $P$ and that maps the curves $A, B, C$ to $A, B, C$ respectively by an orientation-reversing map. The homeomorphism $\alpha$ is the hyperelliptic involution that preserves every simple closed curve (up to isotopy). The automorphism $\beta$ is an orientation-preserving homeomorphism of $S^{3}$ that preserves $V$ and $P$, fixes $T^{-}$ pointwise, and maps $C$ to $C$ and $Y$ to $Y$ by an orientation-reversing map. Also $|A \cap \beta(X)|=2$. The automorphism $\gamma$ preserves $V$ and $P$ and maps the curves $c_{P}$ to $c_{P}$ and $A$ to $A$ by an orientation-reversing map. See Figure 3. Scharlemann [2004] showed that $\mathscr{H}_{2}$ is generated by the isotopy classes $[\alpha],[\beta],[\gamma]$, and $[\delta]$, where $\delta$ is any orientation-preserving homeomorphism of $S^{3}$ such that $\delta(V)=V$ and $v_{P} \cdot v_{\delta(P)}=4$. In this paper we will take $\delta$, as follows. Consider the genus two handlebody $V$ as a regular neighborhood of a sphere, centered at the origin, with three holes. The homeomorphism $\delta$ is a $2 \pi / 3$ rotation of $V$ about the vertical $z$-axis. See Figure 3.

\section{Arc families of reducing spheres on $T^{ \pm}$}

Definition 2. Denote any oriented curve $D$ on $T$ by $\vec{D}$ and the curve oriented in the direction opposite to $\vec{D}$ by $\overleftarrow{D}$

Orient the curves $A, B, C, X, Y, Z$ in such a way that $\delta^{2}(\vec{A})=\delta(\vec{B})=\vec{C}$ and $\delta^{2}(\vec{X})=\delta(\vec{Y})=\vec{Z}$.

Definition 3. For any oriented properly embedded arc $v \subset T^{ \pm}$, we may write $[\nu] \in H_{1}\left(T^{ \pm}, \partial T^{ \pm} ; \mathbb{Z}\right)$ as $a[\mu]+b[\lambda]$ where $\mu=\vec{Z}$ and $\lambda=\vec{B}$ if $\nu \subset T^{-}$, and $\mu=\vec{Y}$ and $\lambda=\vec{C}$ if $v \subset T^{+}$. The slope of $v$ is defined to be $|a / b| \in \mathbb{Q}^{+} \cup \infty$. 
Definition 4. For any reducing sphere $Q$ such that $v_{Q} \neq v_{P}$, let $N\left(Q, T^{ \pm}, a\right)$ denote the number of arcs in $Q \cap T^{ \pm}$of slope $a$.

Definition 5. Up to isotopy, there are natural homeomorphisms $\Omega, \Psi: S^{3} \rightarrow S^{3}$, where $\Omega$ maps $V$ to $W$ and $\vec{A}, \vec{B}, \vec{C}, \vec{X}, \vec{Y}, \vec{Z}$ to $\overleftarrow{X}, \overleftarrow{Y}, \overleftarrow{Z}, \vec{A}, \vec{B}, \vec{C}$, respectively, and $\Psi$ maps $W$ to $W$ and $\vec{A}, \vec{B}, \vec{C}, \vec{X}, \vec{Y}, \vec{Z}$ to $\vec{A}, \vec{B}, \vec{C}, \overleftarrow{X}, \overleftarrow{Y}, \overleftarrow{Z}$, respectively; see Figure 4. Let $\Theta=\Psi \Omega$.

Proposition 1. Let $Q$ be a reducing sphere for $T$ such that $v_{Q} \neq v_{P}$. Then $N\left(Q, T^{-}, a\right)=N\left(Q, T^{+}, 1 / a\right)$.

Proof. Without loss of generality, we may assume that $Q=w(P)$ where $w$ is a word in $\alpha, \beta, \gamma$ and $\delta$.

We claim $\Theta\left(c_{Q}\right)=c_{Q}$. The proof is as follows.

The hyperelliptic involution $\alpha$ preserves the isotopy class of any simple closed curve on $T$. After an isotopy, we may assume that $\alpha\left(c_{Q}\right)=c_{Q}$. Let us write $w$ as $a_{1} a_{2} \cdots a_{n}$ where $a_{i} \in\left\{\alpha, \beta^{ \pm 1}, \gamma, \delta^{ \pm 1}\right\}$. The homeomorphism $\Theta$ satisfies $\Theta \alpha=\alpha \Theta, \Theta \beta=\alpha \beta \Theta, \Theta \gamma=\alpha \gamma \Theta, \Theta \delta=\delta \Theta$, and $\Theta\left(c_{P}\right)=c_{P}$. Then $\Theta\left(c_{Q}\right)=$ $\Theta\left(w\left(c_{P}\right)\right)=\Theta\left(a_{1} a_{2} \cdots a_{n}\left(c_{P}\right)\right)=b_{1} b_{2} \cdots b_{n} \Theta\left(c_{P}\right)$, where $b_{i}$ is $\alpha$ if $a_{i}=\alpha, \alpha \beta$ if $a_{i}=\beta, \alpha \gamma$ if $a_{i}=\gamma$, and $\delta$ if $a_{i}=\delta$. So $b_{1} b_{2} \cdots b_{n} \Theta\left(c_{P}\right)=b_{1} b_{2} \cdots b_{n}\left(c_{P}\right)=$ $a_{1} a_{2} \cdots a_{n}\left(c_{P}\right)=w\left(c_{P}\right)=c_{Q}$.
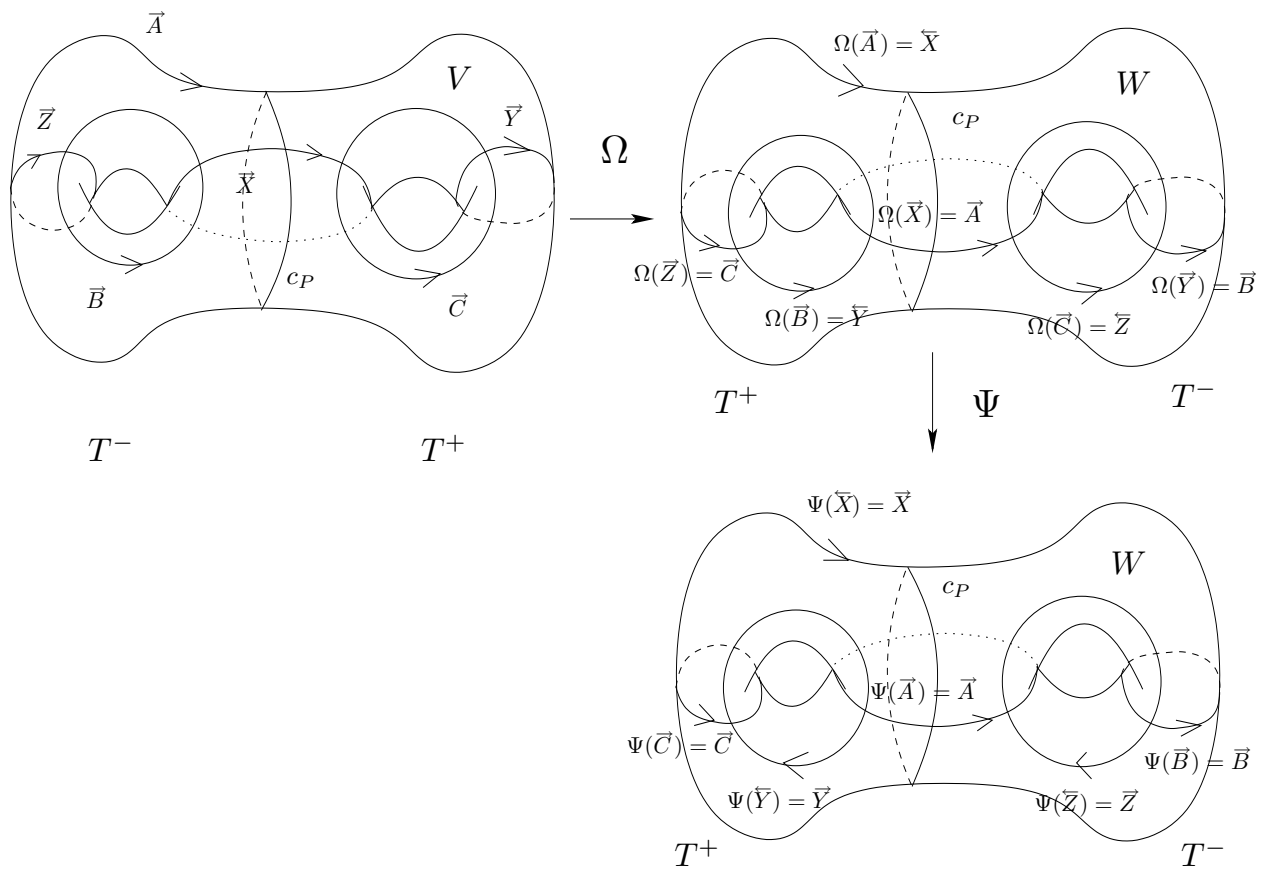

Figure 4. Homeomorphism $\Theta=\Psi \Omega$. 
Since $\Theta$ maps the curves $A, B, C, X, Y, Z$ to $X, Y, Z, A, B, C$, respectively, it takes the $\operatorname{arcs}$ of $c_{Q}$ of slope $a$ on $T^{-}$to the $\operatorname{arcs}$ of $c_{Q}$ of slope $1 / a$ on $T^{+}$.

Definition 6. For any reducing sphere $Q$ for $T$ such that $v_{Q} \neq v_{P}$, let $F_{Q, a}^{ \pm}$denote the arc family of $c_{Q}$ on $T^{ \pm}$of slope $a$.

Lemma 1. Suppose $Q$ is any reducing sphere for $T$ such that $v_{Q} \neq v_{P}$. Then $N\left(Q, T^{-}, 0\right) \neq N\left(Q, T^{-}, \infty\right)$.

Proof. Suppose that $N\left(Q, T^{-}, 0\right)=N\left(Q, T^{-}, \infty\right)=m$. The number $m$ cannot be 0 because the curve $c_{Q}$ must have an arc of slope 0 in either $T^{-}$or in $T^{+}$by [Scharlemann and Thompson 2003, Lemma 4]. By Proposition $1, N\left(Q, T^{+}, 0\right)=$ $N\left(Q, T^{+}, \infty\right)=m$ and $N\left(Q, T^{-}, 1\right)=N\left(Q, T^{+}, 1\right)$. The curve $c_{Q}$ bounds a disc in $V$. So $c_{Q}$ must have a "wave" $\tau$ [Volodin et al. 1974] with respect to one of the curves $Y$ or $Z$. Say it is $Y$, as illustrated below.

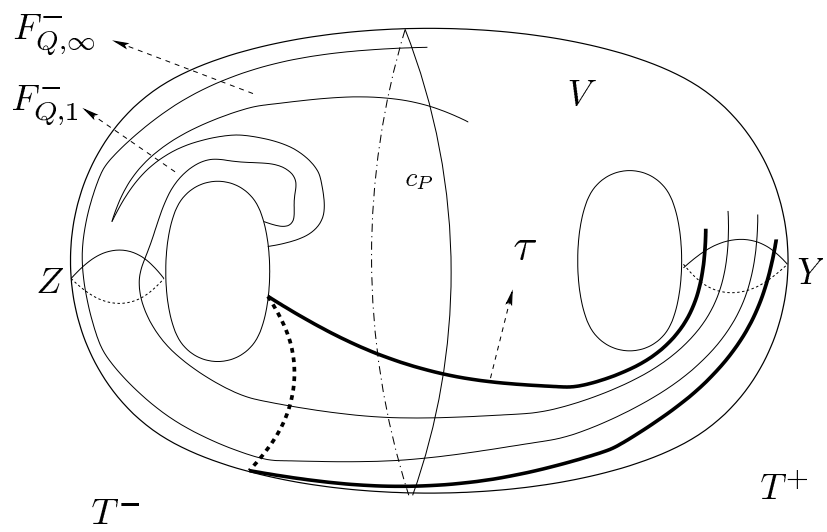

Then the arc $\tau$ of $c_{Q}$ starts at $Y$, goes to $T^{-}$, and then comes back to $Y$ on the same side without touching $Z$. So all the arcs of $c_{Q}$ intersecting $Z$ must intersect the arc on $Y$ that is bounded by ends of $\tau$. Then we get

$$
N\left(Q, T^{-}, \infty\right)+N\left(Q, T^{-}, 1\right)+2 \leq N\left(Q, T^{+}, \infty\right)+N\left(Q, T^{+}, 1\right),
$$

a contradiction.

Notation 1. Let $Q$ be a reducing sphere for $T$.

- If $N\left(Q, T^{-}, 0\right)=n \neq 0$ then $e_{01}, e_{02}, \ldots, e_{0 n}, e_{1 n}, e_{1 n-1}, \ldots, e_{11}$ will denote consecutive end points on $c_{P}$ of the arcs in $F_{Q, 0}^{-}$, where $e_{0 j}$ and $e_{1 j}$ are end points of the same arc; $h_{01}, h_{02}, \ldots, h_{0 n}, h_{1 n}, h_{1 n-1}, \ldots, h_{11}$ will denote consecutive end points on $c_{P}$ of the arcs in $F_{Q, \infty}^{+}$, where $h_{0 j}$ and $h_{1 j}$ are end points of the same arc (the existence of $h_{i j}$ is guaranteed by Proposition 1).

- If $N\left(Q, T^{-}, \infty\right)=m \neq 0$ then $g_{01}, g_{02}, \ldots, g_{0 m}, g_{1 m}, g_{1 m-1}, \ldots, g_{11}$ will denote consecutive end points on $c_{P}$ of the arcs in $F_{Q, \infty}^{-}$, where $g_{0 j}$ are $g_{1 j}$ 
are end points of the same arc; $f_{01}, f_{02}, \ldots, f_{0 m}, f_{1 m}, f_{1 m-1}, \ldots, f_{11}$ will denote consecutive end points on $c_{P}$ of the arcs in $F_{Q, 0}^{+}$, where $f_{0 j}$ and $f_{1 j}$ are end points of the same arc.

- If $N\left(Q, T^{-}, 1\right)=p \neq 0$ then $k_{01}, k_{02}, \ldots, k_{0 p}, k_{1 p}, k_{1 p-1}, \ldots, k_{11}$ will denote consecutive end points on $c_{P}$ of the arcs in $F_{Q, 1}^{-}$where $k_{0 j}$ and $k_{1 j}$ are end points of the same arc; $l_{01}, l_{02}, \ldots, l_{0 p}, l_{1 p}, l_{1 p-1}, \ldots, l_{11}$ will denote end points on $c_{P}$ of the arcs in $F_{Q, 1}^{+}$, where $l_{0 j}$ and $l_{1 j}$ are end points of the same arc.

Lemma 2. Let $Q$ be a reducing sphere for $T$ such that

$$
N\left(Q, T^{-}, 0\right)=n>N\left(Q, T^{-}, \infty\right)=m>N\left(Q, T^{-}, 1\right)=0 .
$$

Then $\left\{f_{i j} \mid i=0,1\right.$ and $\left.j=1, m\right\} \subseteq\left\{e_{i j} \mid i=0,1\right.$ and $\left.j=2, \ldots, n-1\right\}$.

Proof. Suppose the contrary, as illustrated below.

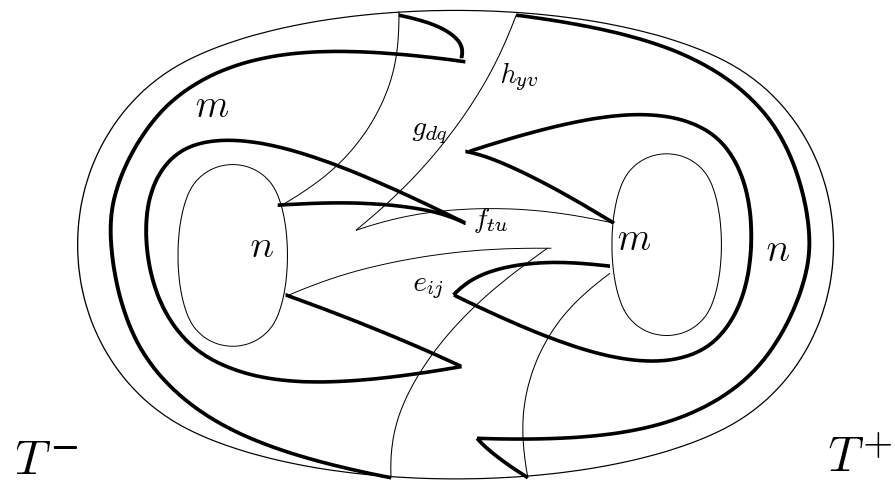

Then $c_{Q}$ does not have a "wave" $\tau$ [Volodin et al. 1974] with respect to the curve $Y$ or the curve $Z$. Therefore $c_{Q}$ cannot bound a disc in $V$, a contradiction.

Proposition 2. Let $v$ and $\tilde{v}$ be any two distinct vertices of $\Gamma$ such that $v \cdot \tilde{v} \neq 4$. Then there exists unique vertex $u$ of $\Gamma$ such that

(i) $u \cdot v=4$,

(ii) $u \cdot \tilde{v}<v \cdot \tilde{v}$, and

(iii) $u \cdot \tilde{v}<v^{\prime} \cdot \tilde{v}$ for any vertex $v^{\prime}$ of $\Gamma$ such that $v^{\prime} \neq u$ and $v^{\prime} \cdot v=4$.

Moreover, there is at most one vertex $v^{\prime \prime}$ of $\Gamma$ satisfying $v \cdot v^{\prime \prime}=4$ and $u \cdot \tilde{v}<$ $v^{\prime \prime} \cdot \tilde{v} \leq v \cdot \tilde{v}$. In this case $v^{\prime \prime} \cdot u=4$.

The proposition is illustrated below. 


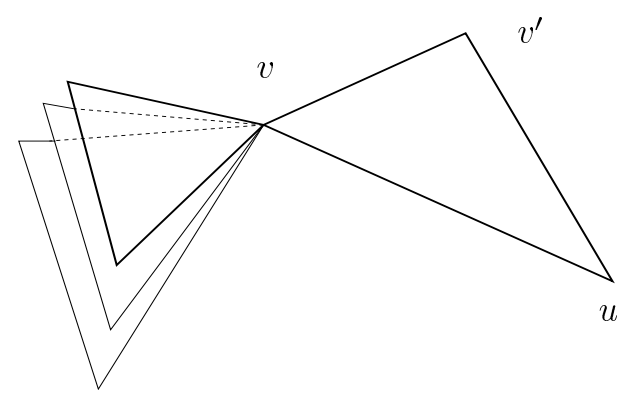

- $\tilde{v}$

Proof. Let $v$ and $\tilde{v}$ be any two vertices of $\Gamma$ such that $v \neq \tilde{v}$ and $v \cdot \tilde{v} \neq 4$. Since the group $\mathscr{H}_{2}$ is transitive on the vertices of $\Gamma$, we may assume that $v=v_{P}$ and $\tilde{v}$ is a vertex of $\Gamma$ such that $\tilde{v} \neq v_{P}$ and $v_{P} \cdot \tilde{v} \neq 4$. Then some word $w$ in $\alpha, \gamma, \beta$ and $\delta$ has $w\left(c_{P}\right) \in \tilde{v}$. Let $Q$ denote the reducing sphere $w(P)$. Since $Q$ is not isotopic to $P$ there must be some arcs in $c_{Q} \cap T^{ \pm}$. By [Scharlemann 2004, Lemma 4] there is an arc of $c_{Q}$ of slope 0 either on $T^{-}$or on $T^{+}$. Suppose it is on $T^{-}$. Let $e_{i j}$, $g_{d q}, k_{r s}, f_{t u}, h_{y v}, l_{w z}$ denote the end points of the $\operatorname{arcs}$ of $c_{Q} \cap T^{ \pm}$as in Notation 1. Possible cases for the arc families in $c_{Q} \cap T^{ \pm}$and their configurations, up to the action of a power of $\beta$, are the following:

Case I. If

$$
\begin{aligned}
N\left(Q, T^{-}, 0\right) & =m, & N\left(Q, T^{-}, 1 / k\right) & =a, \\
N\left(Q, T^{-}, \infty\right) & =0, & N\left(Q, T^{-}, 1 /(k+1)\right) & =b,
\end{aligned}
$$

where $k \geq 1$, then

$$
\begin{aligned}
N\left(Q, T^{+}, \infty\right) & =m, & N\left(Q, T^{+}, k\right) & =a, \\
N\left(Q, T^{+}, 0\right) & =0, & N\left(Q, T^{+}, k+1\right) & =b
\end{aligned}
$$

by Proposition 1. Scharlemann in [2004, Lemma 5] constructs a reducing sphere $R$ satisfying (i) and (ii) (that is, $v_{R} \cdot v_{P}=4$ and $v_{R} \cdot v_{Q}<v_{P} \cdot v_{Q}$ ). We will show that up to isotopy the reducing sphere $R$ also satisfies (iii). Scharlemann's reducing sphere will be given explicitly in the various cases of the proof. Let $n=a+b$.

Case I.A: $n \neq 0$. Let us label end points of the arcs in $c_{Q} \cap T^{+}$of slope different from $\infty$ as $d_{1}, d_{2}, \ldots, d_{2 n}$. Then it is not hard to show $\left\{e_{i j}\right\} \nsubseteq\left\{d_{i}\right\}$ by an argument similar to the proof of Lemma 2.

Case I.A.1: $\left\{d_{i}\right\} \nsubseteq\left\{e_{i j}\right\}$. See the figure below. Set $p=\left|\left\{e_{i j}\right\} \cap\left\{h_{i j}\right\}\right| / 2$ then $1 \leq p<m$. Consider the curve $\xi$ shown in the figure. It is easy to see that $\xi$ bounds a disc in $V$ and a disc in $W$. So $\xi$ is the intersection of a reducing sphere $S$ with $T$. Denote $\xi$ by $c_{S}$. The reducing sphere $S$ satisfies $v_{S} \cdot v_{Q} \leq\left|c_{S} \cap c_{Q}\right|=$ $2(n-m+2 p)<2(n+m)=v_{P} \cdot v_{Q}$ and $v_{S} \cdot v_{P}=4$. 


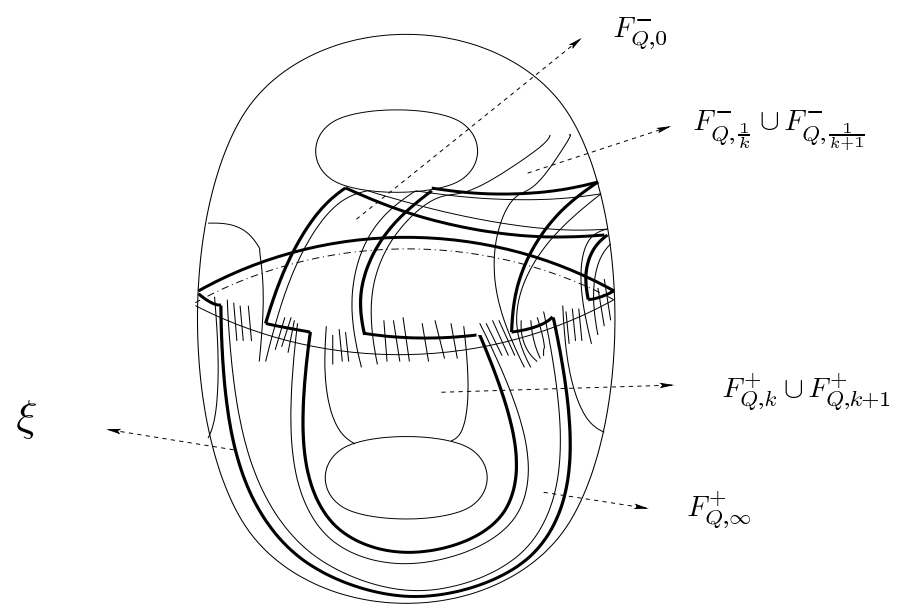

Claim 1. $v_{S} \cdot v_{Q}=\left|c_{S} \cap c_{Q}\right|$.

Claim 2. $v_{\beta^{i}(S)} \cdot v_{Q}, v_{\beta^{i} \gamma(S)} \cdot v_{Q}>2(n+m)$ for $i \neq 0$.

Proof of Claim 1. It suffices to show that there is no bigon on $T$ formed by the curves $c_{S}$ and $c_{Q}$. We may assume that $c_{S}$ intersects $c_{Q}$ in a neighborhood $N \subseteq T$ of $c_{P}$ where $N \cap(B \cup Z \cup C \cup Y)=\varnothing$. The neighborhood $N$ has two boundary components $N^{-}$and $N^{+}$. Say $N^{ \pm} \subset T^{ \pm}$. The set $c_{S} \cap N$ consists of four $\operatorname{arcs} v_{1}$, $v_{2}, v_{3}, v_{4}$. Assume that end points of the $\operatorname{arcs} v_{1}, v_{2}, v_{3}$, and $v_{4}$ on $N^{-}$are lined up consecutively as $N^{-} \cap v_{1}, N^{-} \cap v_{2}, N^{-} \cap v_{3}$, and $N^{-} \cap v_{4}$. The curve $c_{S}$ has two $\operatorname{arcs} a_{1}$ and $a_{2}$ on $T^{-}$of slope 0 and two $\operatorname{arcs} b_{1}$ and $b_{2}$ on $T^{+}$of slope $\infty$. Assume that $v_{i} \cap a_{1} \neq \varnothing$ for $i=1,2$ and $v_{1} \cap b_{1} \neq \varnothing$. See Figure 5. There are eight regions $D_{1}, \ldots, D_{8}$ on $N$ that can contain a vertex of a bigon. The regions $D_{1}, \ldots, D_{8}$ are shown in Figure 5. Any bigon should contain two of them. After an isotopy, we may assume that $\alpha\left(c_{Q}\right)=c_{Q}$ and $\alpha\left(c_{S}\right)=c_{S}$. Then $\alpha\left(D_{i}\right)=D_{i+2}$ for $i=1,2$, and $\Theta\left(\left\{D_{i} \mid i=1, \ldots, 4\right\}\right)=\left\{D_{i} \mid i=5, \ldots, 8\right\}$ (see Definition 5 for $\Theta)$. So it is enough to check if $D_{i}$ is a part of a bigon for $i=1,2$.

$D_{1}$ : The region $D_{1}$ is part of a region $\widetilde{D}_{1}$ in $T$ whose four consecutive sides are $x$, $a_{1}, y$, and $x^{\prime}$, where $y \in F_{Q, 0}^{-}$and $x, x^{\prime} \in F_{Q, k}^{+} \cup F_{Q, k+1}^{+}$. See Figure 6(a). If $\widetilde{D}_{1}$ is a bigon then $v_{Q} \cdot v_{P}<2(n+m)$, a contradiction.

$D_{2}$ : - If $b=0$ then $a \neq 0$. Then $D_{2}$ is part of a region $\widetilde{D}_{2}$ whose five sides are $x, \underset{\widetilde{D}}{a_{1}}, y, y^{\prime}, x^{\prime}$ where $x, x^{\prime} \in F_{Q, \infty}^{+}$and $y, y^{\prime} \in F_{Q, 1 / k}^{-}$. See Figure 6(b). If $\widetilde{D}_{2}$ is a bigon then $v_{Q} \cdot v_{P}<2(n+m)$, a contradiction.

- If $a, b \neq 0$ then $D_{2}$ is part of a region $\widetilde{D}_{2}$ whose five sides are $x, a_{1}, y, y^{\prime}$, $x^{\prime}$ where $x, x^{\prime} \in F_{Q, \infty}^{+}, y \in F_{Q, 1 /(k+1)}^{-}$and $y^{\prime} \in F_{Q, 1 / k}^{-}$. See Figure 6(c). If $\widetilde{D}_{2}$ is a bigon then $v_{Q} \cdot v_{P}<2(n+m)$, a contradiction.

By the cases above, $v_{S} \cdot v_{Q}=\left|c_{S} \cap c_{Q}\right|$. 


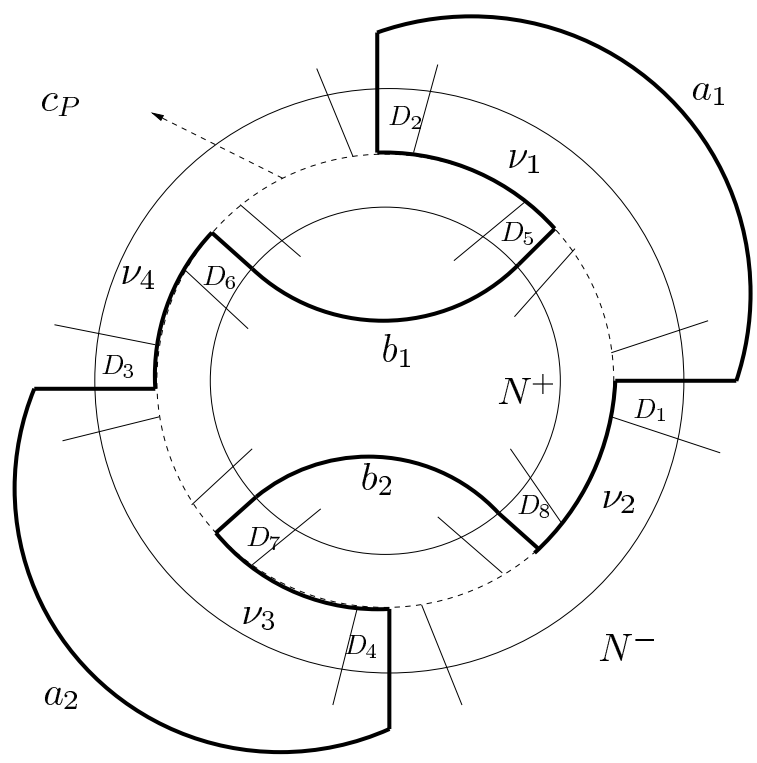

Figure 5

Figure 7 shows the intersection of a reducing sphere $R^{\prime}$ with the surface $T$. Notice that $R^{\prime} \in v_{\gamma S}$ and $v_{S} \cdot v_{\gamma S}=4$. By an argument similar to the proof of Claim 1 we can show that $v_{R^{\prime}} \cdot v_{Q}=\left|c_{R^{\prime}} \cap c_{Q}\right|=4 k b+4(k-1) a+2 m+2 n=$ $v_{\gamma S} \cdot v_{Q} \geq 2 m+2 n$.

Proof of Claim 2. We will do the calculation for $i= \pm 1$. The general case is similar. We may assume that $\beta^{i}\left(c_{S}\right)$ and $\beta^{i} \gamma\left(c_{S}\right)$ intersect $c_{Q}$ in a neighborhood $N$ described in the proof of Claim 1. By an argument similar to the proof of Claim 1 , we get

- $v_{\beta(S)} \cdot v_{Q}=4 p+2 m+6 n>2(n+m)$. See Figure $8(\mathrm{a})$.

- $v_{\beta^{-1}(S)} \cdot v_{Q}=6 m+2 n-4 p>2(n+m)$. See Figure 8(b).

- $v_{\beta \gamma(S)} \cdot v_{Q}=4 k b+4(k-1) a+4 m+2 n+2 p>2(n+m)$. See Figure 9(a).

- $v_{\beta^{-1} \gamma(S)} \cdot v_{Q}=4 k b+4(k-1) a+6 m+6 n-4 p>2(n+m)$. See Figure 9(b).

This implies that the vertex $v_{R}=v_{S}$ and satisfies the conditions of Proposition 2.

Case I.A.2: $\left\{d_{i}\right\} \subseteq\left\{e_{i j}\right\}$. See Figure 10. Set $p=\left|\left\{e_{0 j}\right\} \cap\left\{h_{0 j}\right\}\right|$. Then $0<p \leq m-n$. Either $p<m-n-p$ or $m-n-p<p$. Assume $p<m-n-p$. Consider the curve $\xi$ shown in Figure 10. The curve $\xi$ is an intersection of a reducing sphere $S$ with $T$. Denote $\xi$ by $c_{S}$. Notice that $v_{S} \cdot v_{P}=4$.

By an argument similar to the proof of Case I.A.1, we get 


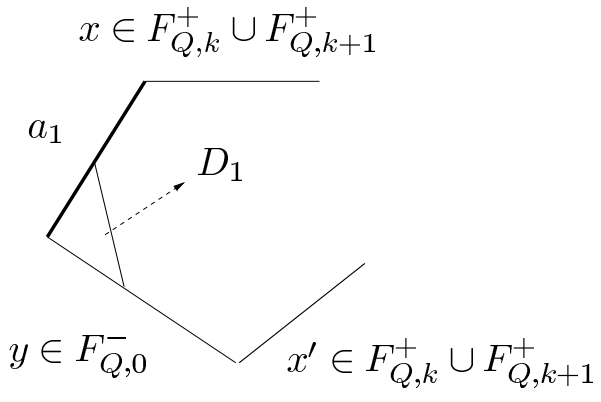

$(a)$

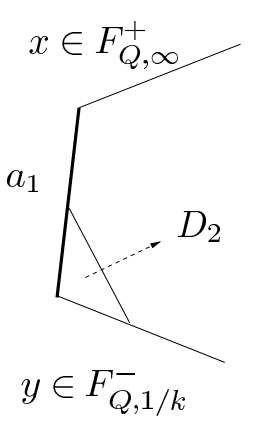

(b)

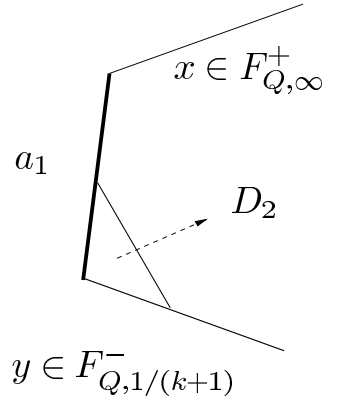

(c)

Figure 6

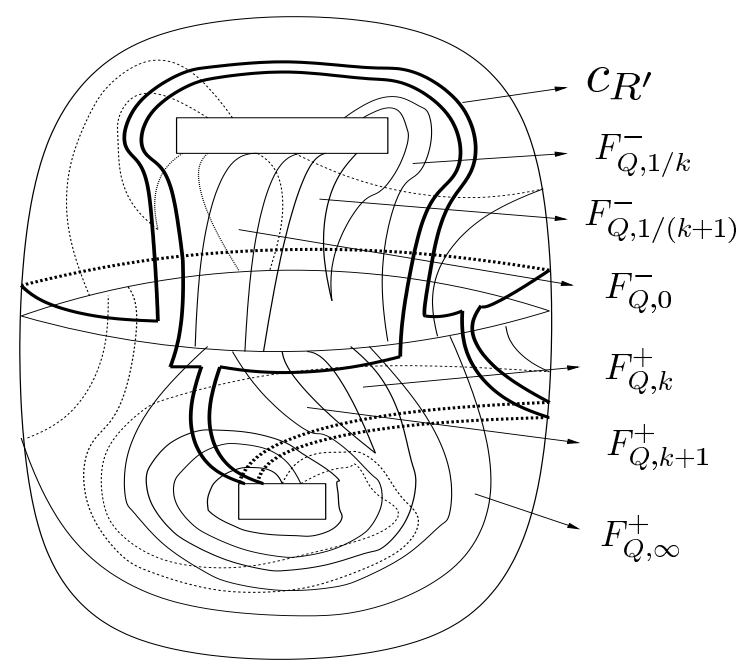

Figure 7 


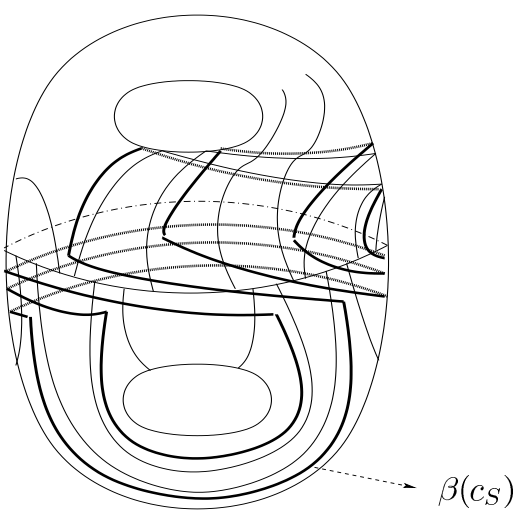

(a)

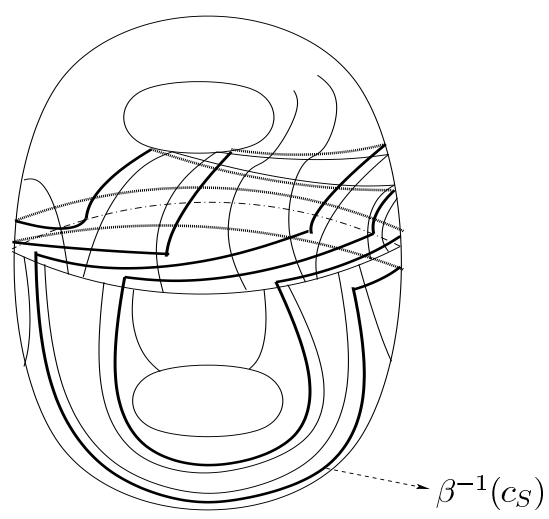

(b)

\section{Figure 8}

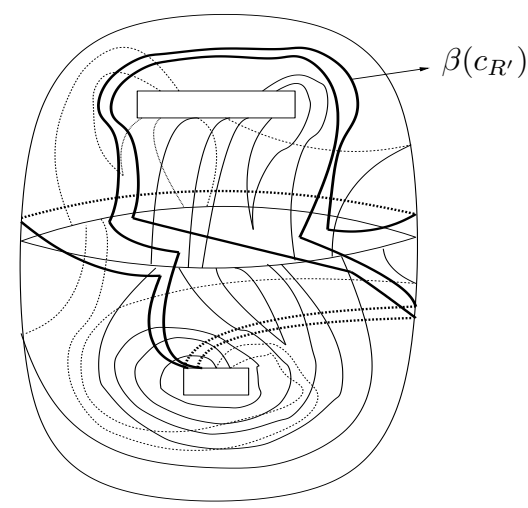

(a)

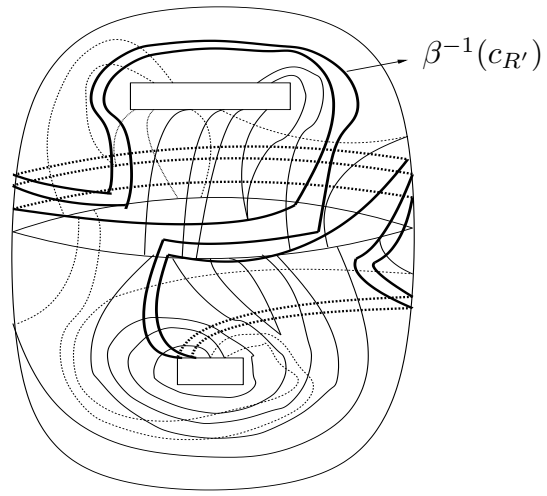

(b)

\section{Figure 9}

- $v_{S} \cdot v_{Q}=\left|c_{S} \cap c_{Q}\right|=2(m-n-2 p)<v_{P} \cdot v_{Q}=2(n+m)$;

- $v_{S} \cdot v_{\gamma(S)}=4$

- $v_{\gamma(S)} \cdot v_{Q}=4 k b+4(k-1) a+2(m+n) \geq 2(m+n)$ (see Figure 11);

- $v_{\beta^{i}(S)} \cdot v_{Q}, v_{\beta^{i} \gamma(S)} \cdot v_{Q}>2(n+m)$ for $i \neq 0$.

This implies that the vertex $v_{R}=v_{S}$ and satisfies the conditions of Proposition 2.

Case I.B: $n=0$. This is a special case of Case I.A.2.

Case II: $N\left(Q, T^{-}, 0\right)=m$ and $N\left(Q, T^{-}, \infty\right)=n \neq 0=N\left(Q, T^{-}, 1\right)$. In this case, $N\left(Q, T^{+}, 0\right)=n$ and $N\left(Q, T^{+}, \infty\right)=m \neq 0=N\left(Q, T^{+}, 1\right)$ by Proposition 1. 


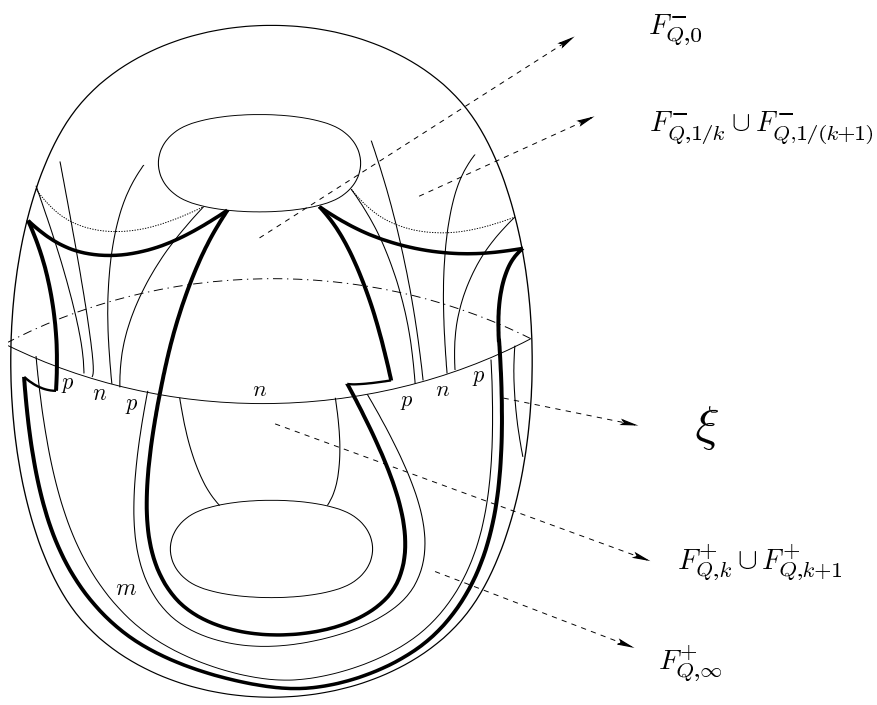

Figure 10

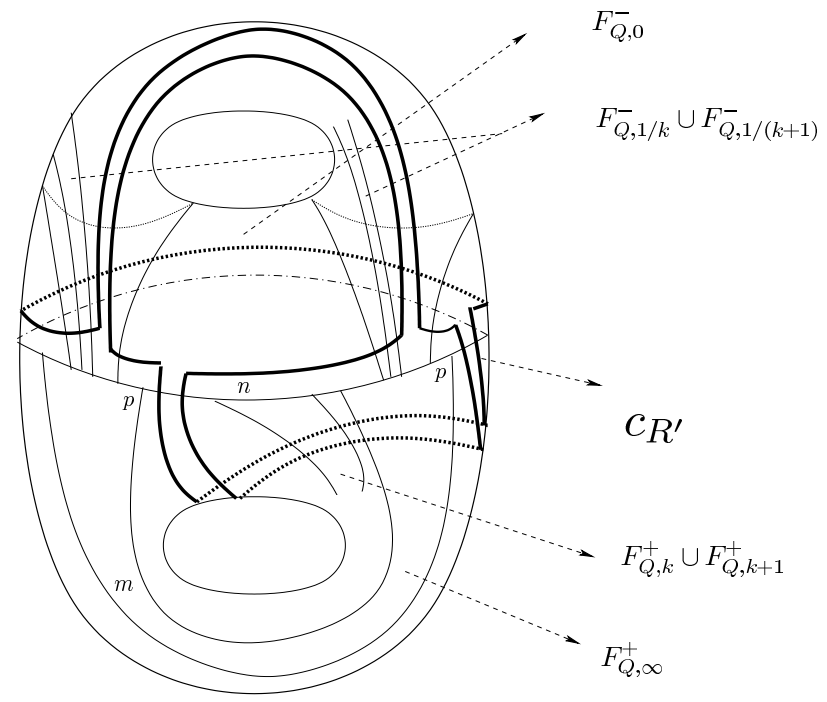

Figure 11. The curve $c_{R^{\prime}}$ in the figure is $R^{\prime} \cap T$ for some reducing sphere $R^{\prime}$ for $T$ satisfying $R^{\prime} \in v_{\gamma S}$.

By Lemma $1, m \neq n$. Suppose $m<n$. By Lemma 2,

$$
\left\{e_{i j} \mid i=0,1 \text { and } j=1, \ldots, m\right\} \subseteq\left\{f_{i j} \mid i=0,1 \text { and } j=2, \ldots, n-1\right\} .
$$

By the argument in [Scharlemann 2004, Lemma 5], we get two nonisotopic reducing spheres for $T$ that satisfy (i) and (ii). Let us call $S$ the one having an arc on 


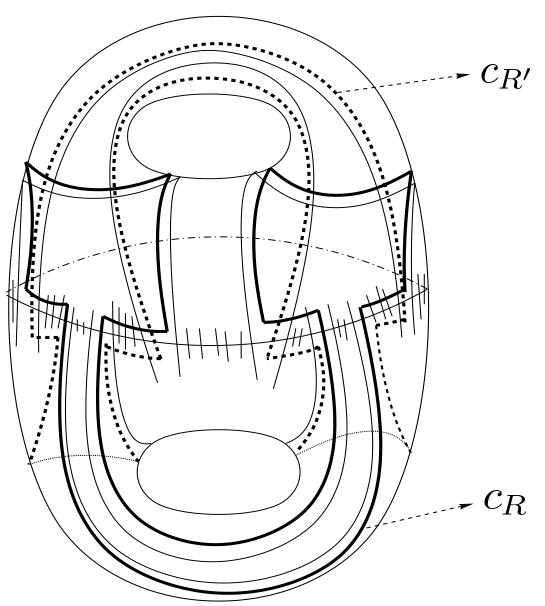

Figure 12

$T^{-}$of slope 0 and $S^{\prime}$ the one having an $\operatorname{arc}$ on $T^{+}$of slope 0 . Figure 12 shows the intersections of two reducing spheres $R$ and $R^{\prime}$ with $T$. It is easy to see that $R \in v_{S}$ and $R^{\prime} \in v_{S^{\prime}}$.

Let $p=\left|\left\{g_{0 j}\right\} \cap\left\{f_{0 j}\right\}\right|$. Then $0<p \leq m-n$. Either $p<m-n-p$ or $m-n-p<p$. Assume $p<m-n-p$. Then by an argument similar to the proof of Case I.A.1, we can show that $2 n+2 m=v_{P} \cdot v_{Q}>v_{R} \cdot v_{Q}=2 n-2 m>v_{R^{\prime}} \cdot v_{Q}=2(n-m-2 p)$, $v_{R} \cdot v_{R^{\prime}}=4$ and $v_{\beta^{i}(R)} \cdot v_{Q}, v_{\beta^{i}\left(R^{\prime}\right)} \cdot v_{Q}>2 n+2 m$ for $i \neq 0$.

Case III: $N\left(Q, T^{-}, 0\right)=m, N\left(Q, T^{-}, \infty\right)=n$, and $N\left(Q, T^{-}, 1\right)=p$ where $m, n, p \neq 0$. In this case, $N\left(Q, T^{+}, 0\right)=n, N\left(Q, T^{+}, \infty\right)=m, N\left(Q, T^{+}, 1\right)=p$ by Proposition 1. By Lemma $1, m \neq n$. Say $m>n$.

The curves $A, B, C$, and $c_{P}$ divide $T$ into four punctured discs $T_{f}^{-}, T_{b}^{-}, T_{f}^{+}$, and $T_{b}^{+}$, where $T_{f}^{-} \cup T_{b}^{-}=T^{-}$and $T_{f}^{+} \cup T_{b}^{+}=T^{+}$. This division also gives two pairs of pants $T_{f}^{-} \cup T_{f}^{+}=P_{f}$ and $T_{b}^{-} \cup T_{b}^{+}=P_{b}$. Let $c_{f}=P_{f} \cap c_{P}$ and $c_{b}=P_{b} \cap c_{P}$.

Let $K$ be a reducing sphere intersecting the interior of $T^{-}$in a simple closed curve parallel to $c_{P}$. The reducing sphere $K$ divides $T$ into two parts. Denote the one containing the curve $B$ by $t^{-}$and the one containing the curve $C$ by $t^{+}$. Let $c_{K}^{f}=T_{f}^{-} \cap K$ and $c_{K}^{b}=T_{b}^{-} \cap K$.

Suppose that

$$
\begin{aligned}
F_{Q, 0}^{-} \cap t^{-} \cap A & =F_{Q, 1}^{-} \cap t^{-} \cap A=\varnothing, \\
\left|F_{Q, \infty}^{-} \cap\left(c_{K}^{f} \backslash A\right)\right| & =\left|F_{Q, \infty}^{-} \cap\left(c_{K}^{b} \backslash A\right)\right|=\left|F_{Q, \infty}^{-} \cap t^{-} \cap A\right|=n
\end{aligned}
$$

and that $k_{01}^{\prime}, k_{02}^{\prime}, \ldots, k_{0 p}^{\prime}, e_{01}^{\prime}, e_{02}^{\prime}, \ldots, e_{0 m}^{\prime}$, and $g_{01}^{\prime}, g_{02}^{\prime}, \ldots, g_{0 n}^{\prime}$ are consecutive intersection points of the arcs in $F_{Q, 1}^{-}, F_{Q, 0}^{-}$and $F_{Q, \infty}^{-}$with $c_{K}^{f}$, respectively. Locate 


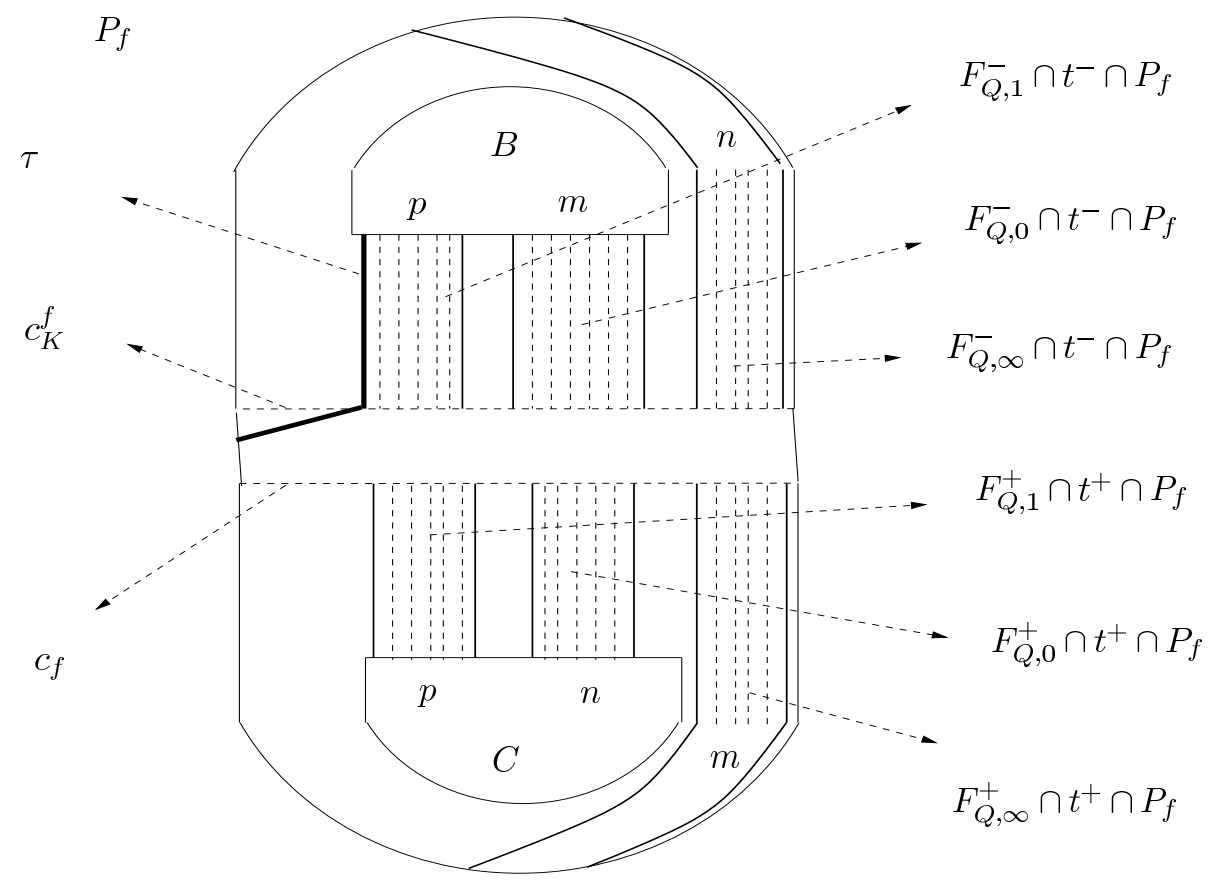

Figure 13

arcs of $c_{Q}$ on $T^{+}$so that

$$
\begin{aligned}
\left|F_{Q, \infty}^{+} \cap\left(c_{P}^{f} \backslash A\right)\right| & =\left|F_{Q, \infty}^{+} \cap\left(c_{P}^{b} \backslash A\right)\right|=\left|F_{Q, \infty}^{+} \cap A\right|=m, \\
\left|F_{Q, 0}^{+} \cap A\right| & =\left|F_{Q, 1}^{+} \cap A\right|=0 .
\end{aligned}
$$

Suppose that $l_{01}, \ldots, l_{0 p}, f_{01}, \ldots, f_{0 n}$, and $h_{01}, \ldots, h_{0 m}$ are consecutive intersection points of the arcs in $F_{Q, 1}^{+}, F_{Q, 0}^{+}$, and $F_{Q, \infty}^{+}$with $c_{f}$, respectively. Suppose $\tau$ is an $\operatorname{arc}$ in $F_{Q, 1}^{-}$whose intersection with $c_{K}^{f}$ is $k_{01}^{\prime}$. Suppose $\tau \cap\left(t^{+} \backslash T^{+}\right) \cap A \neq \varnothing$. See Figure 13. By applying a power of $\beta$, we can assume $2 \leq\left|c_{Q} \cap A \cap\left(t^{+} \backslash T^{+}\right)\right|<$ $2(p+n+m)$. By the argument in [Scharlemann 2004, Lemma 5], we get two nonisotopic reducing spheres for $T$ that satisfy (i) and (ii). Let us call $S$ the one having an $\operatorname{arc}$ on $T^{-}$of slope 0 and $S^{\prime}$ the one having an $\operatorname{arc}$ on $T^{+}$of slope 0 .

The figures below show intersections of two reducing spheres $R, R^{\prime}$ with $T$. It is easy to see that $R \in v_{S}$ and $R^{\prime} \in v_{S^{\prime}}$.

Case III.A: $\left\{g_{i j}\right\} \subseteq\left\{h_{i j}\right\}$. See Figure 14. Let $x=\left|\left\{h_{i j}\right\} \cap\left\{k_{i j}\right\}\right| / 2$. Arguing as in Case I.A.1, we get $2(n+m+p)=v_{P} \cdot v_{Q}>v_{R^{\prime}} \cdot v_{Q}=2(m+p-n)>v_{R} \cdot v_{Q}=$ $2(m+p-n-2 x), v_{R} \cdot v_{R^{\prime}}=4$ and $v_{\beta^{i}(R)} \cdot v_{Q}, v_{\beta^{i}\left(R^{\prime}\right)} \cdot v_{Q}>v_{P} \cdot v_{Q}$ for $i \neq 0$.

Case III.B: $\left\{g_{i j}\right\} \cap\left\{h_{i j}\right\} \neq \varnothing$ and $\left\{g_{i j}\right\} \cap\left\{f_{i j}\right\} \neq \varnothing,\left\{e_{i j}\right\} \cap\left\{h_{i j}\right\}=\varnothing$. See Figure 15. Let $x=\left|\left\{k_{i j}\right\} \cap\left\{h_{i j}\right\}\right| / 2$. Arguing as in Case I.A.1, we get $2(n+m+p)=$ 


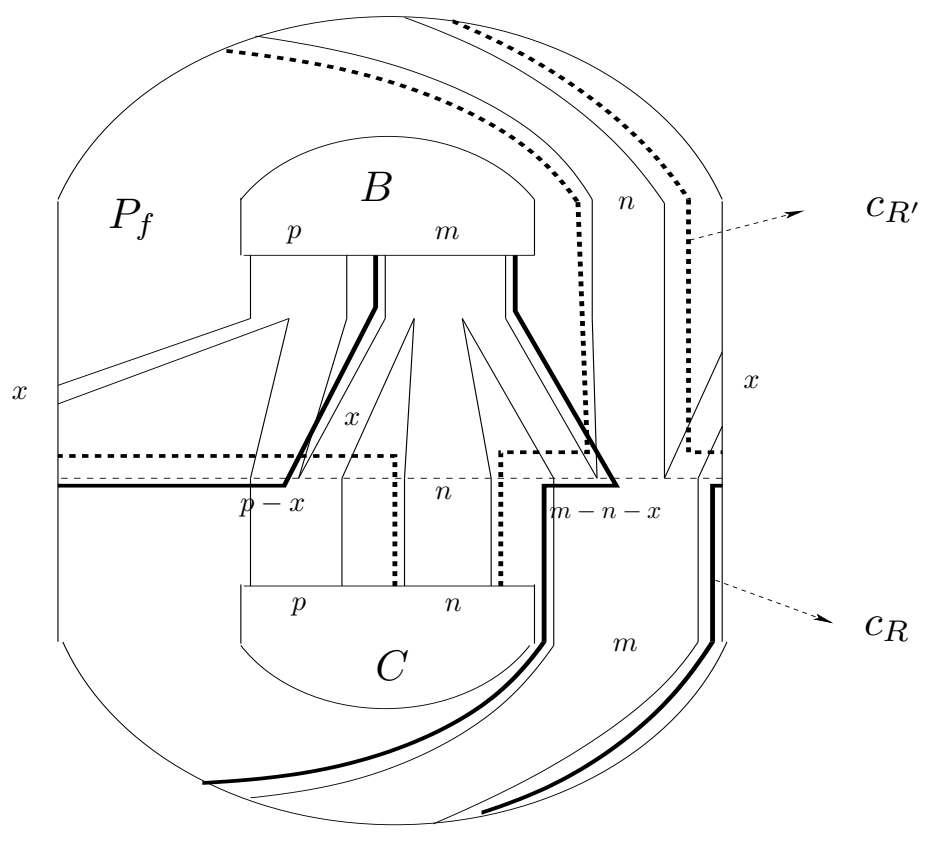

Figure 14

$v_{P} \cdot v_{Q}>v_{R^{\prime}} \cdot v_{Q}=2(p+n-m+2 x)>v_{R} \cdot v_{Q}=2(p+n-m), v_{R} \cdot v_{R^{\prime}}=4$, and $v_{\beta^{i}(R)} \cdot v_{Q}, v_{\beta^{i}\left(R^{\prime}\right)} \cdot v_{Q}>v_{P} \cdot v_{Q}$ for $i \neq 0$.

Case III.C: $\left\{g_{i j}\right\} \cap\left\{h_{i j}\right\} \neq \varnothing,\left\{g_{i j}\right\} \cap\left\{f_{i j}\right\} \neq \varnothing$, and $\left\{e_{i j}\right\} \cap\left\{h_{i j}\right\} \neq \varnothing$. See Figure 16. Let $x=\left|\left\{f_{i j}\right\} \cap\left\{g_{i j}\right\}\right| / 2$. Arguing as in Case I.A.1, we get $2(n+m+p)=$ $v_{P} \cdot v_{Q}>v_{R^{\prime}} \cdot v_{Q}=2(m-n+2 x+p)>v_{R} \cdot v_{Q}=2(m-n-p+2 x), v_{R} \cdot v_{R^{\prime}}=4$, and $v_{\beta^{i}(R)} \cdot v_{Q}, v_{\beta^{i}\left(R^{\prime}\right)} \cdot v_{Q}>v_{P} \cdot v_{Q}$ for $i \neq 0$.

Case III.D: $\left\{g_{i j}\right\} \cap\left\{f_{i j}\right\} \neq \varnothing,\left\{g_{i j}\right\} \cap\left\{l_{i j}\right\} \neq \varnothing,\left\{e_{i j}\right\} \cap\left\{l_{i j}\right\} \neq \varnothing$, and $\left\{e_{i j}\right\} \cap\left\{h_{i j}\right\}=$ $\varnothing$. See Figure 17. Let $x=\left|\left\{g_{i j}\right\} \cap\left\{l_{i j}\right\}\right| / 2$. Arguing as in Case I.A.1, we get $2(n+m+p)=v_{P} \cdot v_{Q}>v_{R^{\prime}} \cdot v_{Q}=2(p+n+m-2 x)>v_{R} \cdot v_{Q}=2(p+n-m)$, $v_{R} \cdot v_{R^{\prime}}=4$, and $v_{\beta^{i}(R)} \cdot v_{Q}, v_{\beta^{i}\left(R^{\prime}\right)} \cdot v_{Q}>v_{P} \cdot v_{Q}$ for $i \neq 0$.

Case III.E: $\left\{g_{i j}\right\} \cap\left\{f_{i j}\right\} \neq \varnothing,\left\{g_{i j}\right\} \cap\left\{l_{i j}\right\} \neq \varnothing,\left\{e_{i j}\right\} \cap\left\{l_{i j}\right\} \neq \varnothing$, and $\left\{e_{i j}\right\} \cap\left\{h_{i j}\right\} \neq \varnothing$. See Figure 18. Let $x=\left|\left\{g_{i j}\right\} \cap\left\{l_{i j}\right\}\right| / 2$. Arguing as in Case I.A.1, we get $v_{R^{\prime}} \cdot v_{Q}=$ $2(m+n+p-2 x), v_{R} \cdot v_{Q}=2(m+n-p+2 x)$ and $v_{\beta^{i}(R)} \cdot v_{Q}, v_{\beta^{i}\left(R^{\prime}\right)} \cdot v_{Q}>$ $2(n+m+p)$ for $i \neq 0$. So $v_{R^{\prime}} \cdot v_{Q}=v_{R} \cdot v_{Q}$ if and only if $p=2 x$. If $p$ is equal to $2 x$, then by an argument given in the proof of Lemma 2 , we can show that $c_{Q}$ does not bound a disc in $V$. Therefore either $v_{R^{\prime}} \cdot v_{Q}>v_{R} \cdot v_{Q}$ or $v_{R^{\prime}} \cdot v_{Q}<v_{R} \cdot v_{Q}$. Notice that $v_{R} \cdot v_{R^{\prime}}=4$.

Case III.F: $\left\{g_{i j}\right\} \cap\left\{f_{i j}\right\} \neq \varnothing,\left\{g_{i j}\right\} \cap\left\{l_{i j}\right\} \neq \varnothing$, and $\left\{g_{i j}\right\} \cap\left\{h_{i j}\right\} \neq \varnothing$. See Figure 19. Let $x=\left|\left\{g_{i j}\right\} \cap\left\{f_{i j}\right\}\right| / 2$. Arguing as in Case I.A.1, we get $2(n+m+p)=$ 


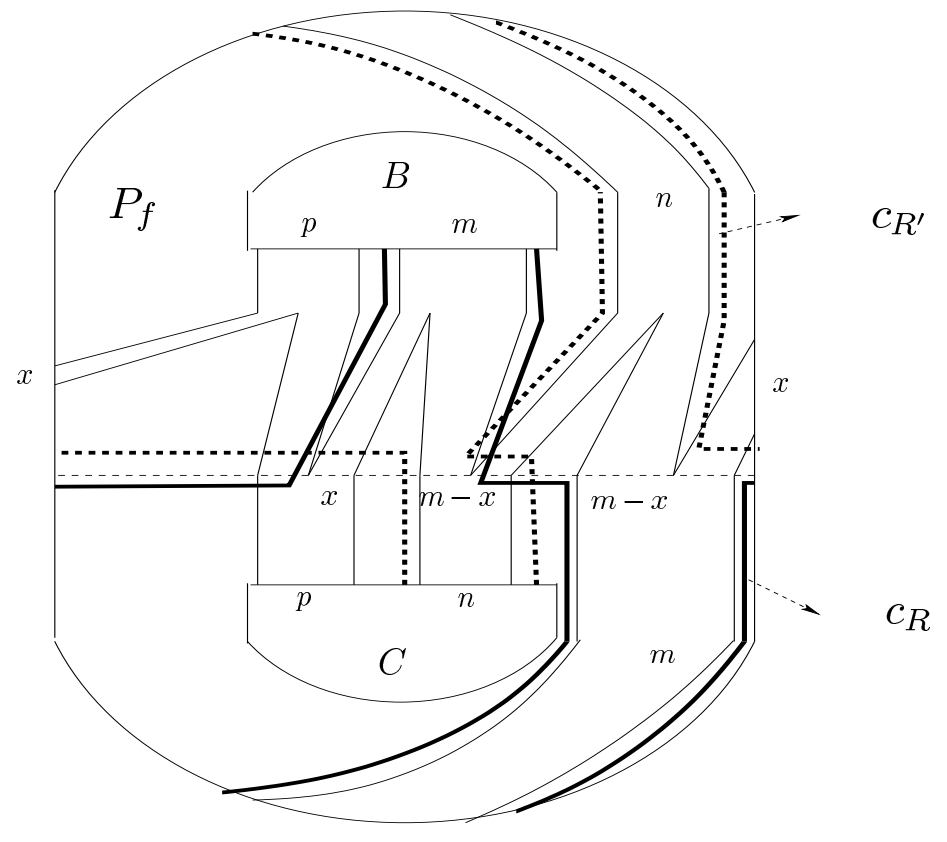

Figure 15

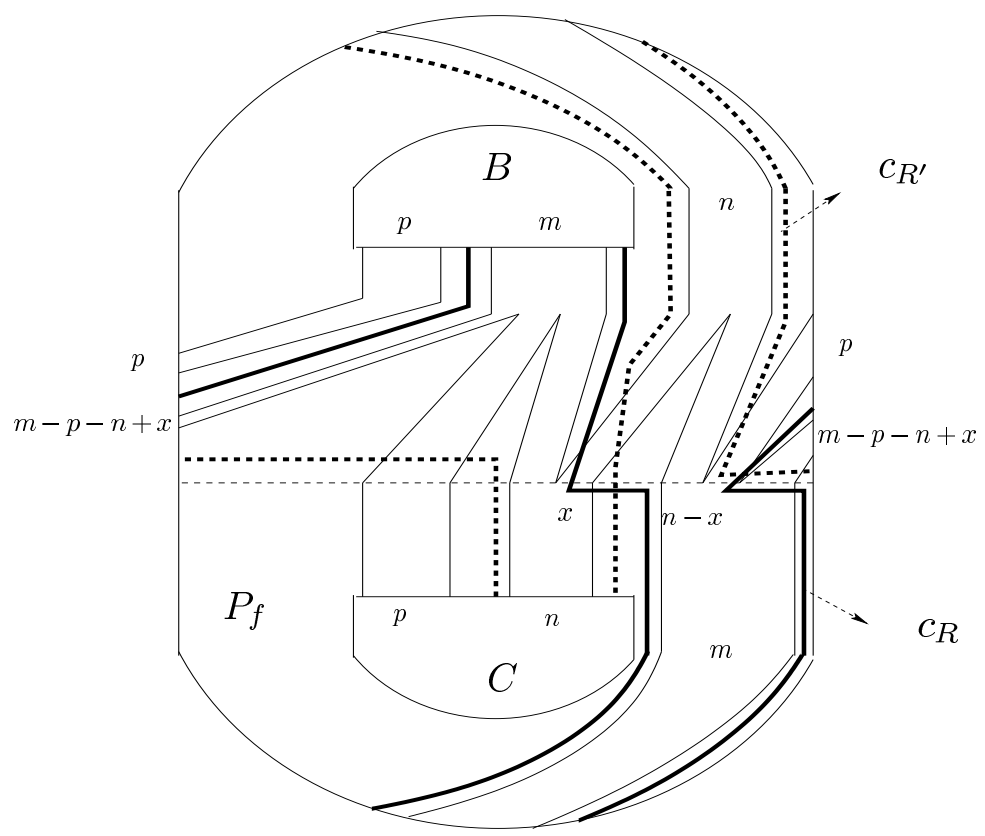

Figure 16 


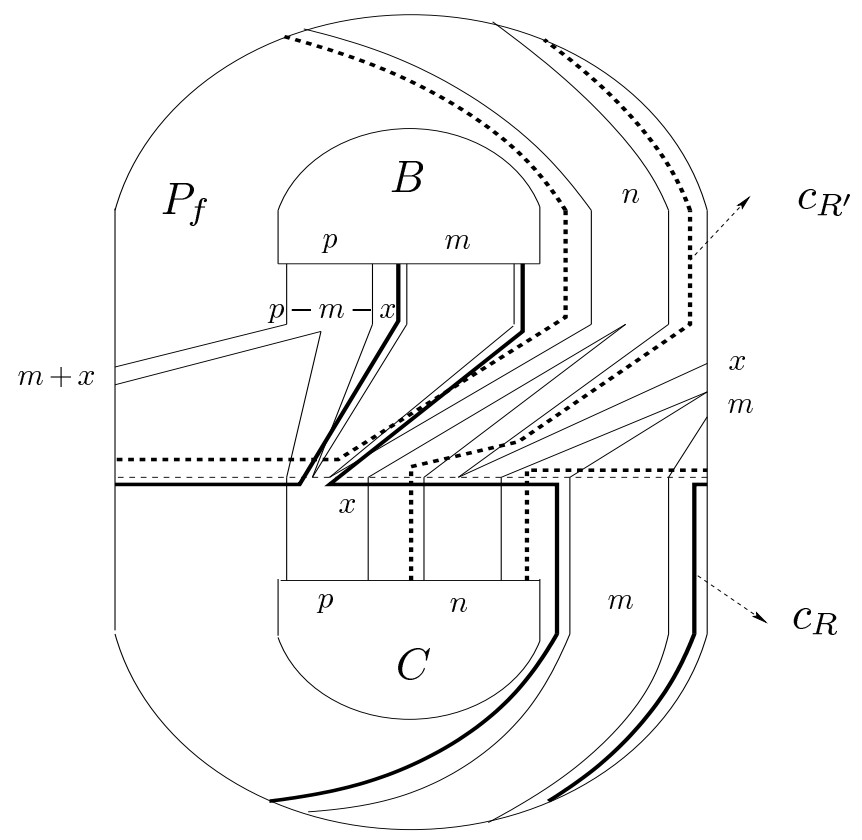

Figure 17

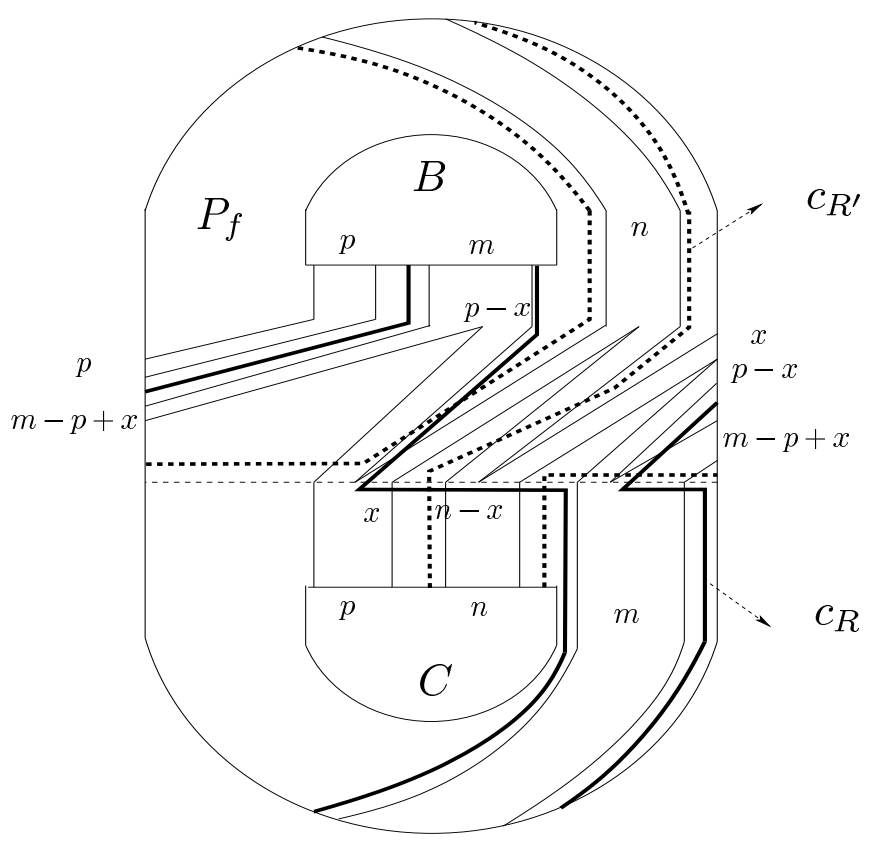

Figure 18 


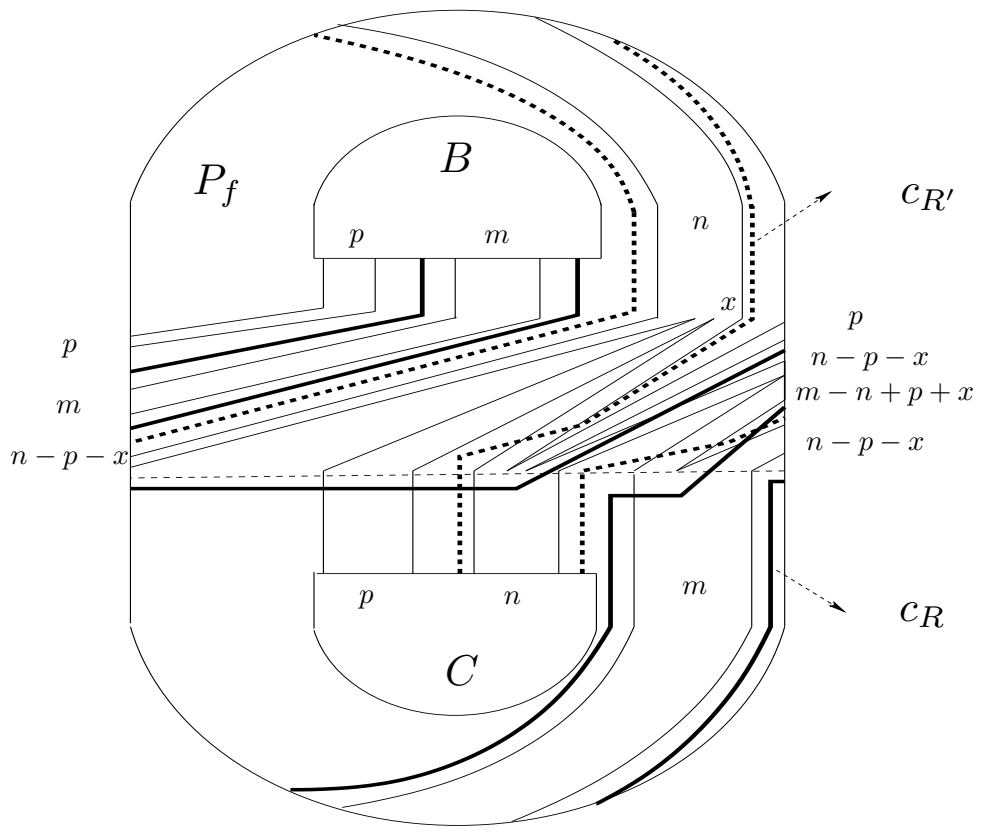

Figure 19

$v_{P} \cdot v_{Q}>v_{R} \cdot v_{Q}=2(m+x+3 p-n+x)>v_{R^{\prime}} \cdot v_{Q}=2(m+x+p-n+x)$, $v_{R} \cdot v_{R^{\prime}}=4$, and $v_{\beta^{i}(R)} \cdot v_{Q}, v_{\beta^{i}\left(R^{\prime}\right)} \cdot v_{Q}>v_{P} \cdot v_{Q}$ for $i \neq 0$.

Case III.G: $\left\{e_{i j}, g_{i j}\right\} \subseteq\left\{l_{i j}\right\}$. See Figure 20. Let $x=\left|\left\{k_{i j}\right\} \cap\left\{l_{i j}\right\}\right| / 2$. Arguing as in Case I.A.1, we get $v_{P} \cdot v_{Q}>v_{R^{\prime}} \cdot v_{Q}=2(p+m-n)>v_{R} \cdot v_{Q}=2(p-m+n)$, $v_{R} \cdot v_{R^{\prime}}=4$, and $v_{\beta^{i}(R)} \cdot v_{Q}, v_{\beta^{i}\left(R^{\prime}\right)} \cdot v_{Q}>v_{P} \cdot v_{Q}$ for $i \neq 0$.

Case III.H: $\left\{g_{i j}\right\} \subseteq\left\{l_{i j}\right\}$ and $\left\{e_{i j}\right\} \cap\left\{h_{i j}\right\} \neq \varnothing$. This case is eliminated by an argument given in proof of Lemma 2 (the curve $c_{Q}$ does not bound a disc in $V$ ).

Case III.I: $\left\{g_{i j}\right\} \cap\left\{l_{i j}\right\} \neq \varnothing$ and $\left\{g_{i j}\right\} \cap\left\{h_{i j}\right\} \neq \varnothing$. After applying $\beta^{-1}$ to $c_{Q}$ we can assume that $c_{Q}$ is as in Figure 21. Let $x=\left|\left\{k_{i j}\right\} \cap\left\{l_{i j}\right\}\right| / 2$ then by arguing as in Case I.A.1, we have $2(n+m+p)=v_{P} \cdot v_{Q}>v_{R} \cdot v_{Q}=2(m-n+3 p-2 x)>$ $v_{R^{\prime}} \cdot v_{Q}=2(m-n+p), v_{R} \cdot v_{R^{\prime}}=4$, and $v_{\beta^{i}(R)} \cdot v_{Q}, v_{\beta^{i}\left(R^{\prime}\right)} \cdot v_{Q}>v_{P} \cdot v_{Q}$ for $i \neq 0$.

\section{A presentation for $\mathscr{H}_{2}$}

We will first prove Theorem 1. Then by using Bass-Serre theory we will prove Theorem 2.

Proof of Theorem 1. Suppose that $\tilde{\Gamma}$ is not a tree. Then there is a nontrivial loop in $\tilde{\Gamma}$. For any loop $\xi$ in $\tilde{\Gamma}$, let $N V(\xi)$ denote the number of vertices of $\xi$. Then 


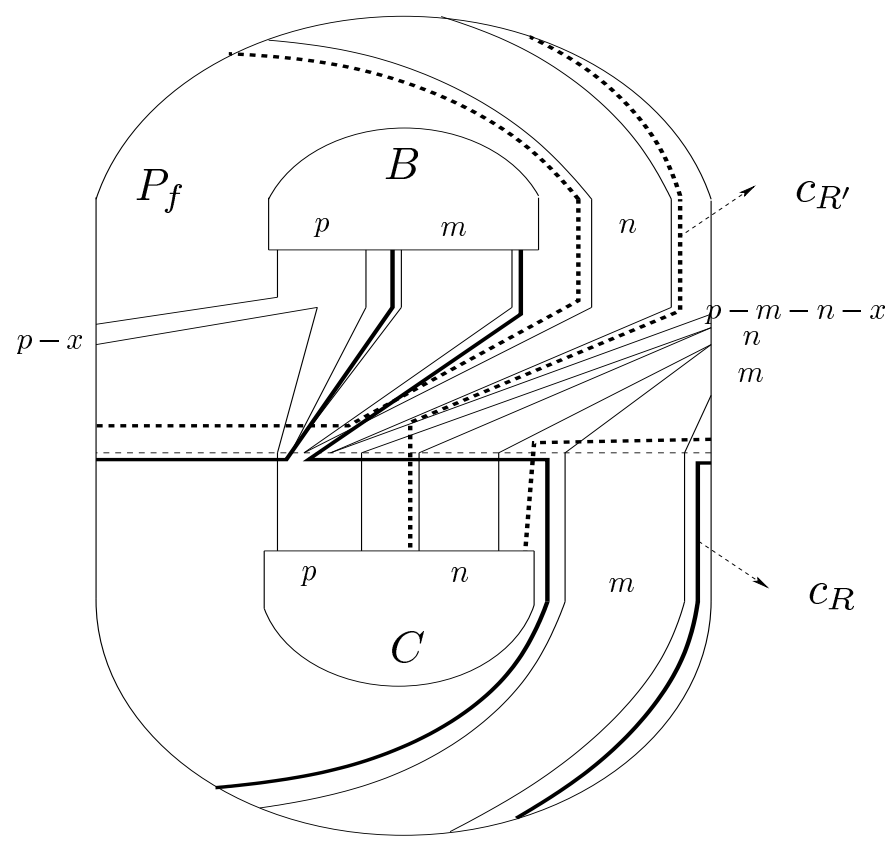

Figure 20

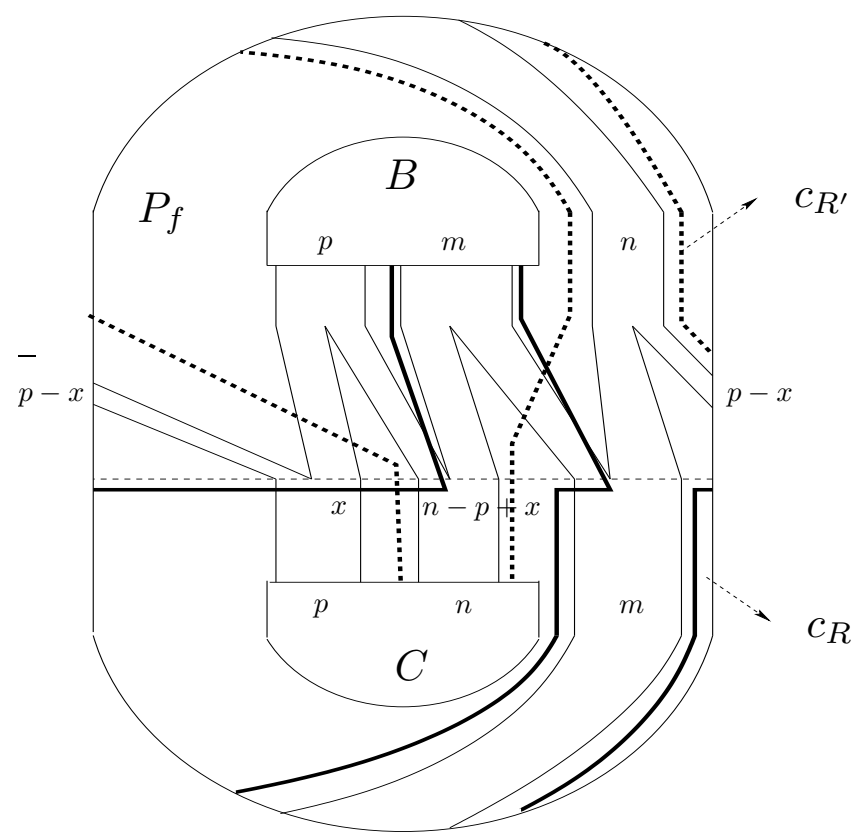

Figure 21 
$\alpha_{0}=\min \{N V(\xi) \mid \xi$ is a nontrivial loop in $\tilde{\Gamma}\}>0$. Since each edge of $\Gamma$ lies on a single 2-simplex, $\alpha_{0} \geq 8$. Let $\xi_{0}$ be a nontrivial loop in $\tilde{\Gamma}$ such that $N V\left(\xi_{0}\right)=\alpha_{0}$. Since $\xi_{0}$ is of minimal length all its vertices are distinct. Let $v_{0}$ be any vertex of $\xi_{0}$, and let $v_{0}, v_{1}, v_{2}, v_{3}, \ldots, v_{\alpha_{0}-1}$ be the consecutive vertices of $\xi_{0}$. We may suppose that $v_{0} \in \Gamma$. Then $v_{0}, v_{2}, v_{4}, \ldots, v_{\alpha_{0}-2}$ are vertices of $\Gamma$, and $v_{k} \cdot v_{k+2}=v_{\alpha_{0}-2} \cdot v_{0}=4$ for $k \in\left\{0,2,4, \ldots, \alpha_{0}-4\right\}$.

We claim $v_{k} \cdot v_{0}<v_{k+2} \cdot v_{0}$ for $k \in\left\{0,2,4, \ldots, \alpha_{0}-4\right\}$. The proof will be by induction on the index $k$, as follows.

If $k=0$, then $v_{0} \cdot v_{0}=0<v_{2} \cdot v_{0}=4$. Assume $v_{k} \cdot v_{0}<v_{k+2} \cdot v_{0}$ for $k \in$ $\left\{0,2, \ldots, \alpha_{0}-6\right\}$. If $v_{k+4} \cdot v_{0} \leq v_{k+2} \cdot v_{0}$, then $v_{k} \cdot v_{k+4}=4$ by Proposition 2 . Since $v_{k} \cdot v_{k+2}=v_{k+2} \cdot v_{k+4}=4$, the vertices $v_{k}, v_{k+2}, v_{k+4}$ form a 2-simplex $\triangle$ in $\Gamma$. Then we get a loop $\xi$ in $\tilde{\Gamma}$ with vertices $v_{0}, v_{1}, \ldots, v_{k}, u, v_{k+4}, v_{k+5}, \ldots, v_{\alpha_{0}-2}$, $v_{\alpha_{0}-1}$, where $u$ is the barycenter of $\triangle$. This contradicts the minimality of $\alpha_{0}$.

By the claim above, we get $v_{0} \cdot v_{\alpha_{0}-4}<v_{0} \cdot v_{\alpha_{0}-2}$. But $4<v_{0} \cdot v_{\alpha_{0}-4}$ and $v_{0} \cdot v_{\alpha_{0}-2}=4$, a contradiction.

Proof of Theorem 2. Let $v_{M}$ be a vertex of $\tilde{\Gamma}$ corresponding to the barycenter of the 2-simplex whose vertices are $v_{P}, v_{\delta(P)}$ and $v_{\delta^{2}(P)}$. Let $E$ be the edge of $\tilde{\Gamma}$ whose vertices are $v_{P}$ and $v_{M}$. Let $H_{P}$ be the subgroup of $\mathscr{H}_{2}$ generated by the elements that stabilize $v_{P}$. Let $H_{M}$ be the subgroup of $\mathscr{H}_{2}$ generated by the elements that preserve $v_{M}$. Let $H_{E}$ be the group of elements of $\mathscr{H}_{2}$ that stabilize the edge $E$.

- Scharlemann in [2004, Lemma 2] presents $H_{P}$ as

$$
\begin{aligned}
H_{P} & =\left\langle[\alpha],[\beta],[\gamma] \mid[\alpha]^{2}=[\gamma]^{2}=[\alpha \gamma]^{2}=\left[\alpha \beta \alpha \beta^{-1}\right]=1,[\gamma \beta \gamma]=[\alpha \beta]\right\rangle \\
& \cong\left(\mathbb{Z} \oplus \mathbb{Z}_{2}\right) \rtimes \mathbb{Z}_{2} .
\end{aligned}
$$

- The subgroup $H_{M}$ fixes the set $\left\{v_{P}, v_{\delta(P)}, v_{\delta^{2}(P)}\right\}$. Therefore

$$
\begin{aligned}
H_{M} & =\langle[\delta],[\alpha],[\gamma]|[\delta]^{3}=[\alpha]^{2}=[\gamma]^{2}=\left[\alpha \delta \alpha^{-1} \delta^{-1}\right]=[\alpha \gamma]^{2}=1, \\
& {\left.[\delta]=\left[\gamma \delta^{2} \gamma\right]\right\rangle } \\
& \left(\mathbb{Z}_{3} \rtimes \mathbb{Z}_{2}\right) \oplus \mathbb{Z}_{2} .
\end{aligned}
$$

- An element $h$ of $\mathscr{H}_{2}$ fixes the sets $\left\{v_{P}\right\}$ and $\left\{v_{\delta(P)}, v_{\delta^{2}(P)}\right\}$ if and only if $h \in H_{E}$. Hence

$$
\begin{aligned}
H_{E} & =\left\langle[\alpha],[\gamma] \mid[\alpha]^{2}=[\gamma]^{2}=[\alpha \gamma]^{2}=1\right\rangle \\
& \cong \mathbb{Z}_{2} \oplus \mathbb{Z}_{2} .
\end{aligned}
$$

The action of $\mathscr{H}_{2}$ on the 2-complex $\Gamma$ induces an action of $\mathscr{H}_{2}$ on the tree $\tilde{\Gamma}$. The subgroups $H_{P}$ and $H_{M}$ are the isotropy subgroups of $\mathscr{H}_{2}$ fixing the vertices $v_{P}$ and $v_{M}$, respectively. By the standard Bass-Serre theory [Serre 2003], the group $\mathscr{H}_{2}$ is thus a free product of the subgroups $H_{P}$ and $H_{M}$ amalgamated over the 
subgroup $H_{E}$ :

$$
\mathscr{H}_{2} \cong H_{P} \underset{H_{E}}{*} H_{M} \cong\left(\mathbb{Z} \oplus \mathbb{Z}_{2}\right) \rtimes \mathbb{Z}_{2} \underset{\mathbb{Z}_{2} \oplus \mathbb{Z}_{2}}{*}\left(\mathbb{Z}_{3} \rtimes \mathbb{Z}_{2}\right) \oplus \mathbb{Z}_{2} .
$$

\section{Acknowledgment}

This paper is a result of my thesis [Akbas 2006]. I am grateful to my advisor, Professor Marc Culler, for his kind supervision and support. I would like to thank Ian Agol for his suggestions for the proofs of Lemmas 1 and 2. I would also like to thank Yo'av Rieck for his helpful conversations.

\section{References}

[Akbas 2006] E. Akbas, A presentation for the automorphisms of the 3-sphere that preserve a genus two Heegaard splitting, PhD thesis, University of Illinois at Chicago, 2006.

[Goeritz 1933] L. Goeritz, "Die Abbildungen der Brezelfläche und der Volbrezel vom Gesschlect 2", Abh. Math. Sem. Univ. Hamburg 9 (1933), 244-259.

[Powell 1980] J. Powell, "Homeomorphisms of $S^{3}$ leaving a Heegaard surface invariant", Trans. Amer. Math. Soc. 257:1 (1980), 193-216. MR 80m:57005 Zbl 0445.57008

[Scharlemann 2004] M. Scharlemann, "Automorphisms of the 3-sphere that preserve a genus two Heegaard splitting”, Bol. Soc. Mat. Mexicana (3) 10:Spec. Iss. (2004), 503-514. MR 2007c:57020 Zbl 1095.57017

[Scharlemann and Thompson 2003] M. Scharlemann and A. Thompson, "Unknotting tunnels and Seifert surfaces", Proc. London Math. Soc. (3) 87:2 (2003), 523-544. MR 2004e:57015 Zbl 1047. 57008

[Serre 2003] J.-P. Serre, Trees, Springer Monographs in Mathematics, Springer, Berlin, 2003. MR 2003m:20032 Zbl 1013.20001

[Volodin et al. 1974] I. A. Volodin, V. E. Kuznecov, and A. T. Fomenko, "The problem of discriminating algorithmically the standard three-dimensional sphere", Russian Math. Surveys 29:5 (1974), 71-172. MR 53 \#9219 Zbl 0311.57001

Received October 19, 2006. Revised February 13, 2008.

EROL AKBAS

Georgia State University

DEPARTMENT OF MATHEMATICS AND STATISTICS

30 PRYOR STREET

ATLANTA, GA 30303

UNited STATES

matexa@langate.gsu.edu 